\title{
Virtual Sensor Based Feedback Active Noise Control For Neonates in NICU
}

\author{
${ }^{1}$ Veena S, Pavithra $\mathrm{S}^{1}$, Lokesha $\mathrm{H}^{1}$ and Narasimhan S. $\mathrm{V}^{1}$ \\ Aerospace Electronics and Systems Division \\ CSIR-National Aerospace Laboratories \\ Bangalore - 560 017, India \\ veenas@nal.res.in
}

\author{
Neha V More ${ }^{2}$ \\ Electronics and Communication Department \\ National Institute of Technology Karnataka \\ Surathkal 575 025, India
}

\begin{abstract}
In this paper, a novel approach based on Feedback Active Noise Control with Virtual sensing has been proposed to reduce the Neonatal Intensive Care Unit (NICU) noise for infants in incubator. In NICU, the noise is due to infant monitoring medical equipments located in the vicinity of incubators. To address this, the algorithms in the literature are based on feedforward approach which requires a good reference to achieve effective attenuation. In an NICU environment, these algorithms require more than one reference microphone, which increases the complexity of the algorithm. This is eliminated by the proposed Feedback Active Noise Control approach as it generates its own reference from the error signal. The baby's ears must fall in the zone of silence (ZOS) to ensure noise reduction at its ears. But the volume of $\mathrm{ZOS}$ is inversely proportional to noise frequency and for frequencies above $1 \mathrm{KHz}$ the ZOS is less than an inch. This constraint is overcome by the virtual sensing technique, which focuses the ZOS at baby's ears. The algorithm is evaluated with noise record from Neonatal Intensive Care Unit and it has resulted in $7 \mathrm{~dB}$ more reduction at the baby's ears compared to existing algorithms.
\end{abstract}

Index Terms - ANC, NICU noise, Incubator ANC, Feedback ANC, Virtual ANC

\section{INTRODUCTION}

Neonatal Intensive Care Unit (NICU) is a place specialized for the care of ill or premature newborn infants, where in they are placed in baby warmers/incubators. For the well being of premature infants, the recommended NICU noise level is a maximum of $50 \mathrm{~dB}$ SPL (sound pressure level). Noise beyond this prescribed level disrupts the child's energy consumption, leading to physiological instability and problems with growth and development. It can lead to permanent cochlear damage, hearing delay, speech delay etc. Most commonly, babies lose the ability to hear the frequencies that they've been constantly exposed to. But NICU becomes noisy due to both external and internal sources. The external noise can be mitigated by following noise reduction protocols ${ }^{1}$. The inside noise is due to equipments like pulse oxy-meter, ventilators etc. placed near the baby and their levels can go more than $70 \mathrm{~dB}$. However, these beeps and alarms from these equipments are very much essential to draw the attention of the nursing staff and hence their levels cannot be reduced.

In view of this, there is a necessity to explore a noise reduction scheme targeting the baby's ears. Two noise reduction schemes based on passive or active control technologies are available in the literature ${ }^{2}$ for consideration. The passive methodology attenuates noise by creating an acoustical seal (like mufflers), which prevents the noise from entering the area of interest, in this case, the baby's ears. This is not a feasible solution as the seals come in contact with baby's skin and its tight fitment applies pressure around baby's ears and hence not acceptable by the medical community. Alternatively, the ANC technology attempts noise attenuation by injecting equal magnitude, anti-phase signal (referred as anti noise) to cancel noise signal by the principle of super position. This technique creates silence packet termed as 'zone of silence' and if it is ensured around baby's ears, sufficient noise reduction can be achieved depending upon the frequency content of the noise signal. Principle wise, this method appears to be a feasible solution.

\section{BACKGROUND AND PROPOSED MODEL}

In all the work carried out so far to reduce incubator noise [3],[4],[5],[6],[7],[8],[9]. a two channel feed forward approach (FFANC) is used. However their performance is based on the reference microphone placement. In an NICU, noise is mainly due to alarms and beeps from monitoring equipments and a single reference may not be sufficient to achieve good performance and increasing the number of references increase the computational complexity. Also, the noise characteristics at the baby's ears may be different from that of the reference. In view of this, FBANC is a better approach than FFANC. Since the beeps and alarm sounds are predictable in nature, FBANC is expected to perform well.

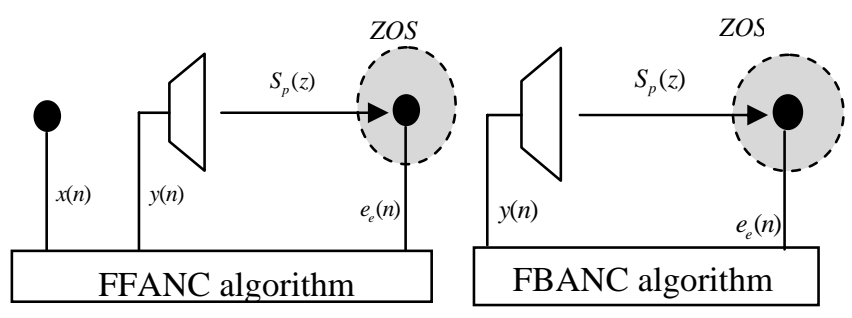

Fig. 1 (a) FFANC setup. (b) FBANC setup.

But, in both the ANC schemes in Figure 1, the noise reduction is observed in zone of silence (ZOS), the volume around the error microphone indicated by shaded area. This volume is 
inversely proportional to the frequency of the noise being attenuated [11]. The ears of the listener have to fall in this zone to perceive the noise reduction. In NICU applications, the microphone cannot be put very close to the ears of the baby and hence ANC may not be an effective solution, unless the ZOS encompasses the ears.

Further, the frequency of NICU sounds plays a major role in deciding the achievable ZOS. For example, ZOS achievable for $1 \mathrm{KHz}$, is about 1 inch as it is one tenth of the wavelength involved [Snyder]. Further, maximum attenuation is achieved at the error microphone, however this reduces as moved away. But from medical requirement and baby's perspective, the error microphone cannot be placed very near to baby's ears and this may result in baby's ear to be excluded from ZOS.

The virtual sensing technique [12] enables the placement of microphone elsewhere from its actual location. This is achieved by considering the transfer function between the actual sensor position and the desired location.

In this paper, it is proposed to combine virtual sensing technique along with FBANC to achieve noise reduction at the baby's ears. The proposed system is evaluated with the actual NICU noise record. The proposed system has resulted in the significant improvement of about $7 \mathrm{~dB}$ compared to FFANC/FBANC approach without virtual sensing.

\section{A. Virtual Microphone Technique}

In ANC based on virtual microphone technique, the objective is to achieve ZOS at baby's ears even when the error microphone is placed away. To accomplish this, the baby's ear location is treated as the virtual location and the adaptive filter weights are updated using the error at this location (virtual error). This error is derived from the error microphone pickup (physical error).

\section{Virtual error estimation}

As shown in Figure 2(a), The estimation of virtual error, $\hat{e}_{v}(n)$ requires path estimates between (i) secondary speaker and physical microphone, $\hat{S}_{p}(z)$ (ii) secondary speaker and virtual microphone, $\hat{S}_{v}(z)$ and (iii) physical and virtual microphones, $\hat{H}(z)$. Microphones are placed at physical and virtual locations and the paths $\hat{S}_{p}(z)$ and $\hat{S}_{v}(z)$ are estimated by injecting zero mean random noise through the loudspeaker. $\hat{H}(z)$ is estimated by picking up the ambient noise by both the microphones. LMS algorithm is used for the estimation of all the three paths. It is important to note that the microphone at virtual location is required for path identification purpose and can be removed thereafter.

(a)

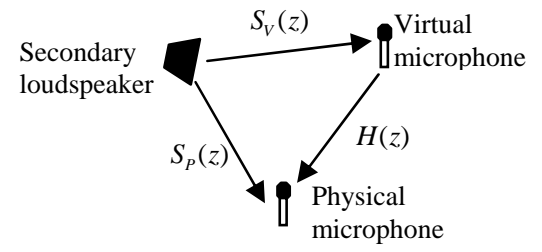

(b)

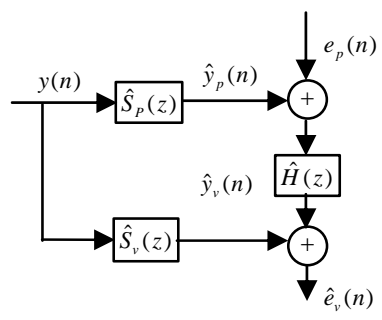

Fig2. (a) Arrangement for estimation of paths. (b) Computation of virtual error. below.

The procedure for estimating the virtual error is given

i. Estimate of the primary disturbance $d_{p}(n)$, at the physical microphone $\hat{d}_{p}(n)$ is calculated using

$$
\begin{aligned}
\hat{d}_{p}(n) & =e_{p}(n)+\hat{y}_{p}(n) \\
& =e_{p}(n)+\hat{S}_{p}(z) * y(n)
\end{aligned}
$$

Where, $\hat{y}_{p}(n)$ is an estimate of secondary disturbance at the physical microphone.

$y(n)$ is the control signal.

$e_{p}(n)$ is the error signal picked up at the physical microphone.

$\hat{S}_{p}(z)$ is the estimate of the physical secondary path.

ii. Primary disturbance at the virtual location $\hat{d}_{v}(n)$ is

$$
\hat{d}_{v}(n)=\hat{H}(z) * \hat{d}_{p}(n)
$$

iii. Virtual error $e_{v}(n)$ is calculated using

$$
\begin{aligned}
e_{v}(n) & =\hat{d}_{v}(n)-\hat{y}_{v}(n) \\
& =\hat{d}_{v}(n)-\hat{S}_{v}(z) * y(n)
\end{aligned}
$$

Where, $\hat{y}_{v}(n)$ is an estimate of secondary

disturbance at the virtual microphone.

$\hat{S}_{v}(z)$ is an estimate of the virtual secondary path.

This error is used for adaptation of adaptive filter coefficients and thus the ZOS is achieved in the virtual location.

\section{B. Model Improvements}

The proposed scheme combines FBANC and Virtual sensing techniques to achieve noise reduction at baby's ears. Here , FBANC derives reference from error microphone signal and thus works independent of noise location. Further, the virtual microphone technique ensures the noise reduction at baby's ears. To account for this, the FBANC algorithm is modified as follows

i. Input signal is generated by virtual error using

$$
\tilde{x}(n)=\hat{e}_{v}(n)+\hat{y}_{p}(n)=\hat{e}_{v}(n)+\hat{S}_{p}(z) * y(n)
$$


Where $\tilde{x}(n)$, is the input generated using virtual error signal.

ii. Weights are adapted using

$$
w_{1}(n+1)=w_{1}(n)+\mu x^{\prime}(n-l) e_{v}(n)
$$

Where, $\quad x^{\prime}(n)=\tilde{x}(n) * \hat{S}_{v}(n)$

Where , $x^{\prime}(n)$ is the filtered input signal that is computed by filtering input signal through an estimate of secondary path at virtual location.

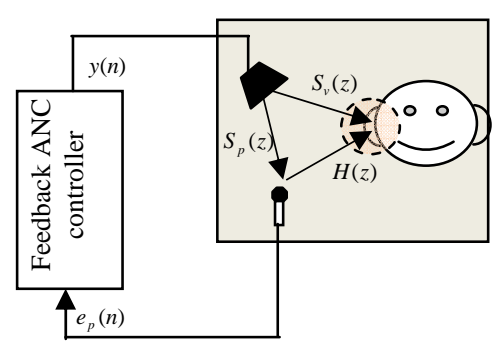

Fig 3. Proposed ANC scheme

\section{LABORATORY AND FIELD MEASUREMENTS}

The proposed ANC scheme was evaluated with the real NICU noise data and the results obtained are presented below.

\section{A. Field measurements}

The noise environment at NICU of St. John's Medical Hospital, Bangalore was taken as a case study. Here, the babies are placed in open type incubator/baby warmers. The noise inside NICU is due to monitoring equipment. The noise signals and the $\mathrm{dB}$ levels were recorded by using M-Audio track recorder and B \& K sound level meter, respectively. These equipments were placed near the baby's ear position at one of the incubators for measurement.

TABLE I

NOISE LEVELS AND FREQUENCY CONTENT OF VARIOUS SOURCES

${ }^{*} \mathrm{dBA}$ scale is used as it matches the ear response of human beings and is the preferred metric for sound level measurements.

\begin{tabular}{l||c|c}
\hline \multicolumn{1}{c||}{ Source } & $\begin{array}{c}\text { Noise level in } \\
\text { dBA }\end{array}$ & $\begin{array}{c}\text { Frequency in } \\
\text { KHz }\end{array}$ \\
\hline \hline $\begin{array}{l}\text { Ventilator } \\
\text { (Dragon) }\end{array}$ & 72 & $2.3,1.2$ \\
\hline $\begin{array}{l}\text { Pulse oxi-meter } \\
\text { beeps }\end{array}$ & 65 & 1.2 \\
\hline $\begin{array}{l}\text { Baby cry } \\
\text { Trolley } \\
\text { movement }\end{array}$ & 68 & 1.3 \\
\hline Human Speech & Upto 80 & 2.2 \\
\hline
\end{tabular}

During the observation period in NICU, the ambient noise was mainly due to the pulse oxy-meter beeps, as this equipment was operational for most of the incubators. This was followed by ventilator beeps and occasional ventilator alarms. Baby cries and human speech was also common in the NICU. The noise levels due to these activities are tabulated in Table I.

\section{B. Laboratory Experiments}

\section{The Experimental setup}

The experimental setup consists of an open type incubator/baby warmer fitted with the loudspeaker and microphone as shown in the Figure 4. The noise recordings obtained from NICU were used for performance analysis of the system.

The algorithms were implemented on a Texas Instruments TMS320C6713 DSP processor. The signal conditioners used are having a cut of frequency of $2000 \mathrm{~Hz}$; hence the ANC has an operating range up to $2000 \mathrm{~Hz}$.



Fig 4. Experimental setup

\section{Experiments}

In FFANC [2], placement of reference plays a key role in achieving a good attenuation. As per literature, the reference should be located near the source of sound. However, in actual NICU the source of noise could be from any of the equipments and placing the reference microphone at appropriate position need to be considered. Hence, experiments were conducted for the following scenarios by placing noise generators at four different places around the incubator. The experiments conducted indicate that multiple references were required to achieve good performance.

The FBANC algorithm was evaluated in a similar environment. The reduction achieved at the error microphone was almost similar to FFANC. Therefore, FBANC can be a better alternative for NICU environment. The noise 
attenuation achieved away from the error microphone is tabulated in Table II.

TABLE II

ZONE OF SILENCE MEASUREMENTS

\begin{tabular}{l||c|c}
\hline $\begin{array}{c}\text { Measurement } \\
\text { location }\end{array}$ & $\begin{array}{c}\text { Ambient noise } \\
\text { level in dBA }\end{array}$ & $\begin{array}{c}\text { Attenuation } \\
\text { in dBA }\end{array}$ \\
\hline \hline $\begin{array}{c}\text { At the error } \\
\text { microphone }\end{array}$ & 60.8 & 9.2 \\
\hline $\begin{array}{c}\text { At a distance of } \\
0.5 \text { inch from } \\
\text { error } \\
\text { microphone }\end{array}$ & 62.9 & 5.4 \\
\hline $\begin{array}{l}\text { At a distance of } \\
1.0 \text { inch from } \\
\text { error } \\
\text { microphone }\end{array}$ & 64.1 & 2.03 \\
\hline $\begin{array}{l}\text { At a distance of } \\
1.5 \text { inch from } \\
\text { error } \\
\text { microphone }\end{array}$ & 63.5 & 1.08 \\
\hline
\end{tabular}

For experiments involving virtual sensors, the distance between the virtual and the physical location was fixed at 1.5 inch, the virtual location being the centre of the baby doll's ears. The proposed algorithm resulted in a reduction of about $8 \mathrm{~dB}$ at this location. The $\mathrm{dB}$ meter reading and the perception test were also in agreement with the computed attenuation. The ZOS measurements were made in both the virtual and physical microphone planes, which are 2 inch squares as shown in Figure 5(b) and Figure 5(c), respectively. The baby's ear experiences a reduction of $8 \mathrm{~dB}$.

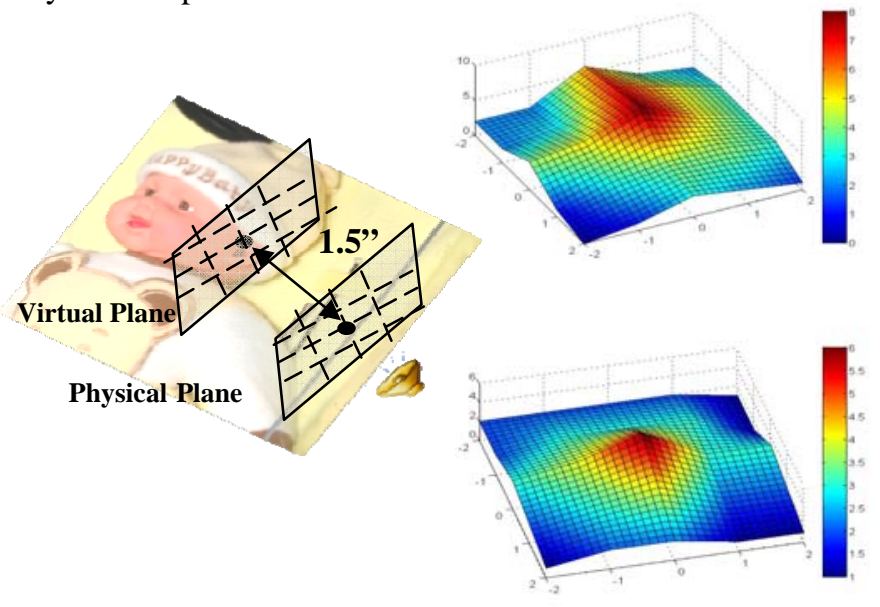

Fig. 5. Measurement of zone of silence for the proposed method. (a) Setup showing virtual and physical planes. (b) Zone of silence measured at virtual plane. (c) Zone of silence measured at physical plane

\section{DISCUSSION OF MODEL PERFORMANCE}

In an NICU environment, the FFANC requires multiple references and this increases the complexity of the algorithm, which is not desirable. The FBANC scheme, which generates reference using error microphone signal is more appropriate for this scenario. However, both the schemes are unable to achieve noise attenuation at baby's ears. The Table II indicates $\mathrm{dBA}$ levels decrease when moved away from the error microphone. At a distance of 1 inch it is almost negligible. Hence, these results are not meaningful from the perspective of creating silence for the baby. The proposed Virtual Sensor based FBANC shows good reduction at the baby's ear location.

\section{CONCLUSION}

In this paper, a novel algorithm based on virtual sensor feedback ANC approach is proposed to be used for NICU noise reduction at infant's ears. The feedback approach dispenses the necessity of reference microphones and thus reduces the computational complexity. The virtual sensing technique shifts the ZOS to the baby's ears instead of error microphone. The proposed algorithm has resulted in $7 \mathrm{~dB}$ more attenuation compared to existing algorithms.

\section{ACKNOWLEDGEMENTS}

The authors are thankful to Dr. Ramesh, ENT Surgeon, St. John's Medical Hospital, Bangalore for showing keen interest and making all the arrangements to visit NICU.

\section{REFERENCES}

1. A. Ramesh, P.N. Suman Rao, G. Sandeep, M. Nagapoornima, V. Srilakshmi, M. Dominic and Swarnarekha, "Efficacy of a Low Cost Protocol in Reducing Noise Levels in the Neonatal Intensive Care Unit”, Indian Journal of Pediatrics, Vol. 76-May, 2009.

2. Sen M. Kuo, Dennis R. Morgan, "Active Noise Control Systems:Algorithms and DSP implementations",(Wiley Series in Telecommunications and Signal processing, New York, 1996).

3. Lichuan Liu, Kaplia Beemanpally and Sen M. Kuo, "Real-time Experiments of ANC Systems for Infant Incubators", Noise Control Engineering Journal, Vol. 60, no. 1, pp. 36-41, Jan-Feb. 2012.

4. Sen M. Kuo, Lichuan Liu, and Shruthi Gujjula, "Development and Application of Audio-Integrated Active Noise Control System for Infant Incubators", Noise Control Engineering Journal, Vol. 58, no. 2, pp. 163-175, March-April 2010.

5. Lichuan Liu, Shruthi Gujjula, Priya Thanigai, and Sen M. Kuo, "Still in Womb: Interuterine Acoustic Embedded Active Noise Control for Infant Incubators", Journal of 
Advances in Acoustics and Vibration, Special Issue on Active Noise Control, Vol. 2008, Article ID 495317, April 2008.

6. Sen M. Kuo and Kapila Beemanpally,"Real-Time Experiments of Active Noise Control Algorithms in Neonatal Intensive Care Units", in Proc. InterNoise 2011 CD, September 2011, pp. 1-9.

7. Kapila Beemanpally, Karunakar R. Pottim and Sen M. Kuo, "Multi-Channel Hybrid Active Noise Control System for Infant Incubators," in Proc. IEEE Int. Conf. on Electro/Information Technology, May 2010, pp. 6976.

8. Lichuan Liu, Shruthi Gujjula, and Sen M. Kuo, "Development and Applications for Active Noise Control System for Infant Incubators," in Proc. 2009 IEEE Int. Conf. on Systems, Man, and Cybernatics, Oct. 2009, pp. 2738-2743.

9. Xun Yu, Shruthi Gujjula, and Sen M. Kuo, "Active Noise Control for Infant Incubators," in Proc. Int. Conf. IEEE Engineering in Medicine and Biology Society, Sept. 2009,pp. 2531-2534.

10. Lichuan Liu, Shruthi Gujjula, and Sen M. Kuo, "Multichannel Real Time Active Noise Control System for Infant Incubators," in Proc. Int. Conf. IEEE Engineering in Medicine and Biology Society, Sept. 2009, pp. 935938.

11. Scott D. Snyder, Active Noise Control Primer, Modern Acoustics and Signal Processing, Springer, 2000.

12. Lichuan Liu, Sen M. Kuo, MengChu Zhou, "Virtual Sensing Techniques and Their Applications”, Proc. 2009 IEEE Int. Conf. on Networking, Sensing and Control, Okayama, Japan, March 26-29, 2009.

13. Yuhsuke Ohta and Akira Sano,"New Direct Adaptive Approach to Multichannel Active Noise Control", Electronics and Communications in Japan, Part 3, Vol. 88, No. 12, 2005. 